Classification

Physics Abstracts

$72.20-77.55$

\title{
Couches minces diélectriques de phosphate d'aluminium
}

\author{
S. Daviéro, C. Avinens, A. Ibanez, J. C. Giuntini et E. Philippot \\ Lab. Physicochimie des Matériaux Solides, UM II, URA D0407, 34095 Montpellier Cedex 05, \\ France
}

(Reçu le 25 mai 1992, révisé le 7 septembre 1992, accepté le 25 septembre 1992)

\begin{abstract}
Résumé. - Réalisation de couches minces de phosphate d'aluminium sur substrat de silicium à partir des précurseurs tributylphosphate et acétylacétonate d'aluminium en solution par la méthode " pyrosol ". La composition chimique des dépôts varie rapidement en fonction des conditions expérimentales. Ces couches minces sont caractérisées par spectrométrie infrarouge et diffraction des RX. Leurs caractéristiques électriques, déterminées par conductivité électrique en courant continu et alternatif, sont nettement différentes de celles du phosphate d'aluminium cristallisé et $s$ expliquent par l'existence de « liaisons pendantes» Al-O et P-O.
\end{abstract}

\begin{abstract}
Aluminium phosphate thin films on silicium substrate have been carried out from tributylphosphate and aluminium acetylacetonate precursors in solution through the " pyrosol » process. It can be observed a large range of chemical analysis in terms of experimental conditions. These thin films have been characterized by X-ray diffraction and infrared spectrometry. Their electrical characteristics, defined from direct current and alternative current measurements, are quite different to those of the crystallized phosphate and can be explained by $\mathrm{P}-\mathrm{O}$ and $\mathrm{Al}-\mathrm{O}$ « dangling bond » existence.
\end{abstract}

\section{Introduction.}

$\mathrm{Au}$ laboratoire, nous nous intéressons particulièrement à la famille des matériaux, $\mathrm{M}^{\mathrm{III}} \mathrm{X}^{\mathrm{V}} \mathrm{O}_{4}$ avec $\mathbf{M}=\mathrm{Al}, \mathrm{Ga}, \mathrm{Fe}$ et $\mathrm{X}=\mathrm{P}$, As. Pendant de nombreuses années, nous avons abordé uniquement l'élaboration et la caractérisation de monocristaux de ces matériaux pour leurs propriétés piézoélectriques. C'est ainsi que nous avons mis au point la cristallogénèse de la berlinite qui est en cours d'industrialisation par SICN (Annecy) et CEPE (Argenteuil) tandis que nous étudions actuellement celle de $\mathrm{GaPO}_{4}$. De plus, ces matériaux présentent des propriétés diélectriques intéressantes, d'où l'idée de les déposer en couches minces isolantes sur des substrats semiconducteurs. Ainsi, le but de ce travail est la réalisation de couches minces diélectriques sur substrat semiconducteur autre que le silicium. Pour ce dernier, on maîtrise parfaitement l'oxydation superficielle du matériau qui conduit à une couche mince isolante de silice. Ce procédé n'étant pas transposable aux semiconducteurs de type III-V par exemple, notre objectif est donc la réalisation de couches de phosphate (ou d'arséniate) d'aluminium (ou de gallium) sur le semiconducteur III-V correspondant par la méthode 
«Pyrosol ». Toutefois, pour des raisons de mise en place de cette nouvelle méthode au laboratoire, nous avons commencé par déposer des couches minces de phosphate d'aluminium sur substrat de silicium.

Nous présenterons, dans un premier temps, les conditions d'élaboration de ces couches minces. Nous exposerons ensuite les différentes caractérisations effectuées sur ces dépôts en nous étendant plus particulièrement sur leurs propriétés électriques que nous essaierons de relier à l'approche structurale.

\section{Synthèse des couches minces.}

La méthode "pyrosol » est une méthode d'élaboration basée sur la thermolyse d'un aérosol réalisé à partir d'une solution contenant les précurseurs du matériau à déposer. Cette solution initiale est pulvérisée à la surface du liquide par une source d'ultrasons. Le brouillard ainsi constitué de fines gouttelettes de taille homogène est propulsé par un gaz vecteur vers le substrat maintenu à une température $T\left({ }^{\circ} \mathrm{C}\right)$. A son contact, le solvant s'évapore et les précurseurs réagissent entre eux à l'état gazeux pour conduire à la formation d'une couche mince.

Ce procédé permet d'obtenir des matériaux pour lesquels il est difficile de trouver des précurseurs gazeux comme ceux nécessaires pour les méthodes de type CVD (chemical vapor deposition). D'autre part, la dissolution des précurseurs permet de les faire réagir à des températures relativement faibles (inférieures à $600^{\circ} \mathrm{C}$ ) et de modifier la concentration des dépôts en modifiant la composition de la solution initiale. Les précurseurs retenus pour la formation de ces dépôts sont le tributylphosphate, TBP, et l'acétylacétonate d'aluminium, AAA, solubilisés dans de l'éthanol.

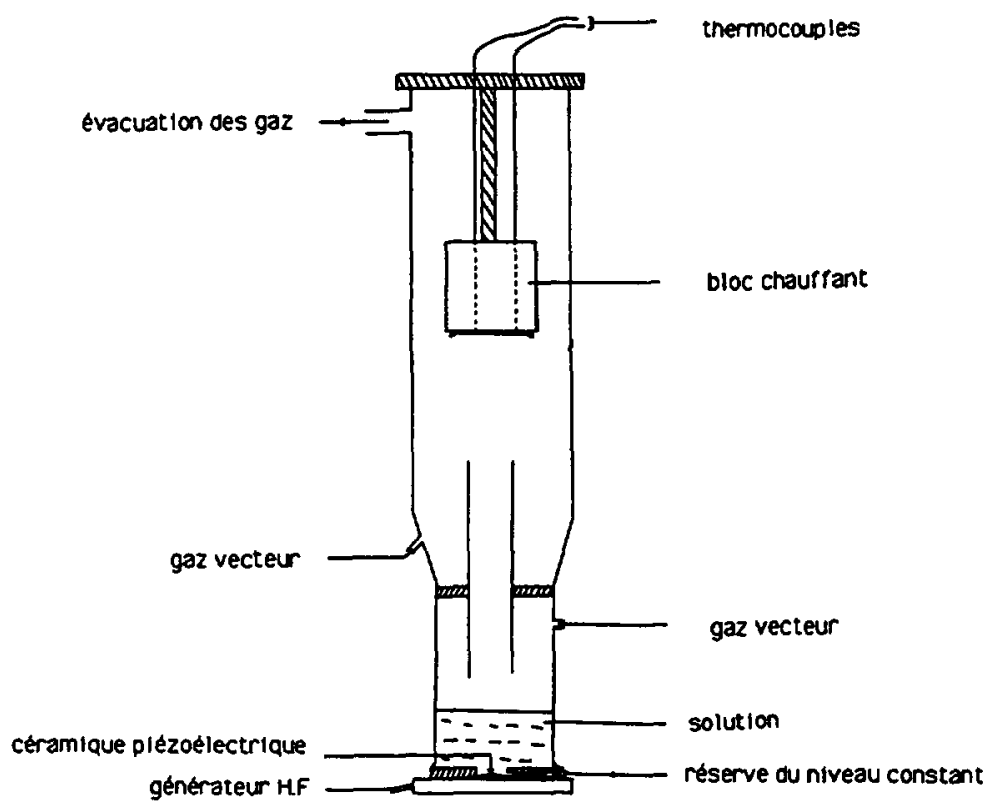

Fig. 1. - Schéma du montage pyrosol.

[Pyrosol equipment.] 
L'élaboration de couches minces par le procédé «pyrosol » peut donc se résumer par le schéma suivant :

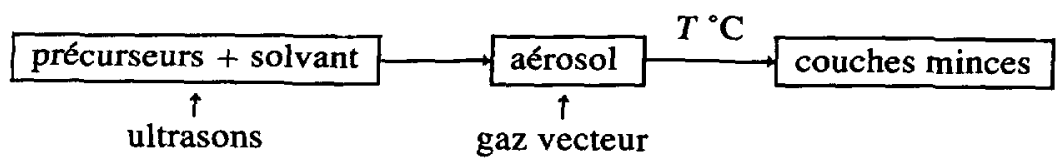

La figure 1 schématise une installation «pyrosol» classique utilisée pour cette étude. La qualité et la composition de la couche mince obtenue dépendent de nombreux paramètres expérimentaux. Sans rentrer dans le détail des conditions de synthèse qui ont déjà été précisées dans un autre article [1], nous pouvons résumer les conditions retenues :

- les solutions initiales ont une concentration fixe de $5 \times 10^{-2}$ mole $^{-1}$ en AAA et la proportion relative des deux précurseurs, exprimée par rapport molaire $\mathrm{Al} / \mathrm{P}$, ne dépend que de la concentration choisie en TBP; $500^{\circ} \mathrm{C}$.

\section{Résultats et discussions.}

3.1 ANALYSE CHIMIQUE DES COUCHES MINCES. - Dans le tableau I, nous avons regroupé les compositions chimiques des dépôts réalisés en fonction des deux paramètres expérimentaux (Al/P et $T_{\mathrm{s}}$ ) que nous venons de définir. Il faut souligner que les dosages de l'aluminium et du phosphore ont tout d'abord été effectués à l'aide d'une sonde EDS (Energy Dispersive Spectroscopy) de précision limitée. A titre de comparaison, le dosage de la forme cristallisée berlinite, $\mathrm{AlPO}_{4}$, donnait un rapport des pourcentages atomiques $\mathrm{Al} / \mathrm{P}=0,93$.

On constate que la teneur en aluminium diminue quand la température augmente avec une très forte variation autour de la valeur $A l / P=1$ pour un domaine étroit de température $\left(\Delta T \# 10^{\circ} \mathrm{C}\right)$. Cette évolution implique donc une régulation très précise de la température $T_{\mathrm{s}}$ lors du dépôt. Ce changement brutal de composition semble dû à la compétition entre deux réactions chimiques. $L$ 'une correspondrait à la réaction proprement dite des deux précurseurs conduisant à la formation de chaînes $[\mathrm{O}-\mathrm{Al}-\mathrm{O}-\mathrm{P}]_{n}$ tandis que l'autre réaction ne ferait

Tableau I. - Composition en fonction des températures de dépôt et de la composition de la solution initiale exprimée en pourcentage molaire de précurseur de l'aluminium.

[Thin film composition in connection with temperatures and initial solution composition (in aluminium molar percent).]

\begin{tabular}{|c|c|c|c|c|c|}
\hline$T\left({ }^{\circ} \mathrm{C}\right)$ & $25 \%$ & $33 \%$ & $\begin{array}{c}50 \% \\
\text { Rapport Al/P }\end{array}$ & $66 \%$ & $75 \%$ \\
\hline 460 & & & 0,69 & 0,80 & \\
450 & & & 0,75 & 0,80 & 5,06 \\
440 & 0,43 & 0,68 & 2,07 & 4,26 & \\
430 & 0,44 & 0,69 & 2,92 & & \\
420 & 0,65 & 0,77 & 3,76 & & \\
410 & 0,74 & 1,35 & & & \\
400 & 2,92 & 3,44 & & & \\
390 & 3,25 & & & & \\
\hline
\end{tabular}


intervenir au craquage que le précurseur AAA entraînant la formation d'enchaînements [O-Al-O-Al $]_{n}$ caractéristiques d'une alumine. En dessous d'une certaine température qui est fonction de la composition de la solution initiale ( $\mathrm{Tab}$. I), la deuxième réaction devient nettement prépondérante et peut être, dans des conditions limites, la seule qui se produit conduisant à l'obtention de couches amorphes d'alumine.

La précision des dosages à la sonde EDS n'étant pas satisfaisante, nous avons ensuite utilisé une sonde WDS (Wave Dispersive Spectroscopy) sur une série d'échantillons obtenus à la même température, $T=450^{\circ} \mathrm{C}$. Par cette technique, il est possible de déterminer la concentration en oxygène de nos dépôts. Nous exprimerons cette teneur sous la forme d'un rapport, $\mathrm{O}_{\text {théor }} / \mathrm{O}_{\text {exp }}$, où $\mathrm{O}_{\text {théor }}$ est calculée par rapport aux teneurs en $\mathrm{Al}$ et $\mathrm{P}$ (\% atomiques) mesurées à la sonde et exprimées sous forme des deux oxydes $\mathrm{Al}_{2} \mathrm{O}_{3}$ et $\mathrm{P}_{2} \mathrm{O}_{5}$ sachant que dans le calcul $2 \mathrm{AlPO}_{4}$ correspondent à $\mathrm{Al}_{2} \mathrm{O}_{3}+\mathrm{P}_{2} \mathrm{O}_{5}$. Nous avons donc $\mathrm{O}_{\text {théor }}=5 / 2[\mathrm{P}]+$ $3 / 2[\mathrm{Al}]$.

Bien que les évolutions des concentrations soient identiques, on observe un écart significatif entre les deux séries de dosage. Les résultats de la sonde WDS sont plus précis et plus fiables car ils tiennent compte de la teneur en oxygène qui n'est estimée que par différence dans le cas de la sonde EDS. Toutefois, par les deux techniques, on note un saut de composition très important pour les couches minces obtenues à partir de solutions initiales de compositions comprises entre 62 et $64 \%$ molaire en AAA, avec au-delà de celles-ci une proportion prépondérante d'aluminium par rapport au phosphore. Une autre caractéristique de ces couches est leur excès en oxygène, $\mathrm{O}_{\text {théor }}$ inférieur à $\mathrm{O}_{\text {exp. }}$. Ces expériences étant effectuées sous courant d'air purifié, nous avons ensuite opéré sous courant inerte d'azote. Dans ces conditions, pour le mélange $50 \%$, on retrouve la même valeur du rapport $\mathrm{Al} / \mathrm{P}$, mais l'excès d'oxygène diminue et le rapport $O_{\text {théor }} / O_{\exp }$ passe de 0,86 à 0,93 .

Tableau II. - Valeurs des compositions des différents dépôts réalisés à $450^{\circ} \mathrm{C}$ en fonction du pourcentage molaire AAA dans la solution initiale.

[Composition of different thin films made at $450{ }^{\circ} \mathrm{C}$ in connection with the AAA molar percent of the initial solution.]

\begin{tabular}{|c|c|c|c|c|c|c|}
\hline $\begin{array}{l}\text { Pourcentage molaire } \\
\text { de AAA en solution }\end{array}$ & $40 \%$ & $50 \%$ & $60 \%$ & $62 \%$ & $64 \%$ & $66 \%$ \\
\hline Sonde EDS Al/P & 0,64 & 0,76 & 0,74 & 0,75 & 3,5 & 2,79 \\
\hline Sonde WDS Al/P & 0,81 & 0,84 & 0,88 & 0,91 & 3,33 & 3,00 \\
\hline $\mathrm{O}_{\text {théor }} / \mathrm{O}_{\text {exp }}$ & 1,00 & 0,86 & 0,87 & 0,88 & 0,86 & 0,95 \\
\hline
\end{tabular}

\subsection{CARACTÉRISATION DES COUCHES MiNCES.}

3.2.1 Epaisseur et aspect des couches au microscope électronique à balayage. - Comme le montrent les photos 1 , les couches présentent un aspect d'autant plus lisse que la composition en aluminium augmente : Al/P 0,81;0,91 et 3,00 (sonde WDS). L'observation transversale de ces mêmes couches permet d'estimer leur épaisseur, généralement comprise entre 0,6 et 1,0 $\mu$, et de mettre en évidence leur structure colonnaire. D'autre part, l'épaisseur peut être mesurée avec une meilleure précision par rugosimétrie. On peut en déduire la vitesse de dépôt, $v$, qui diminue quand la concentration en phosphore augmente dans les couches minces $(v \approx 0,5 \mu \mathrm{m} / \mathrm{h}$ pour $\mathrm{Al} / \mathrm{P}>1, v \approx 0,2 \mu \mathrm{m} / \mathrm{h}$ pour $\mathrm{Al} / \mathrm{P}<1)$. 

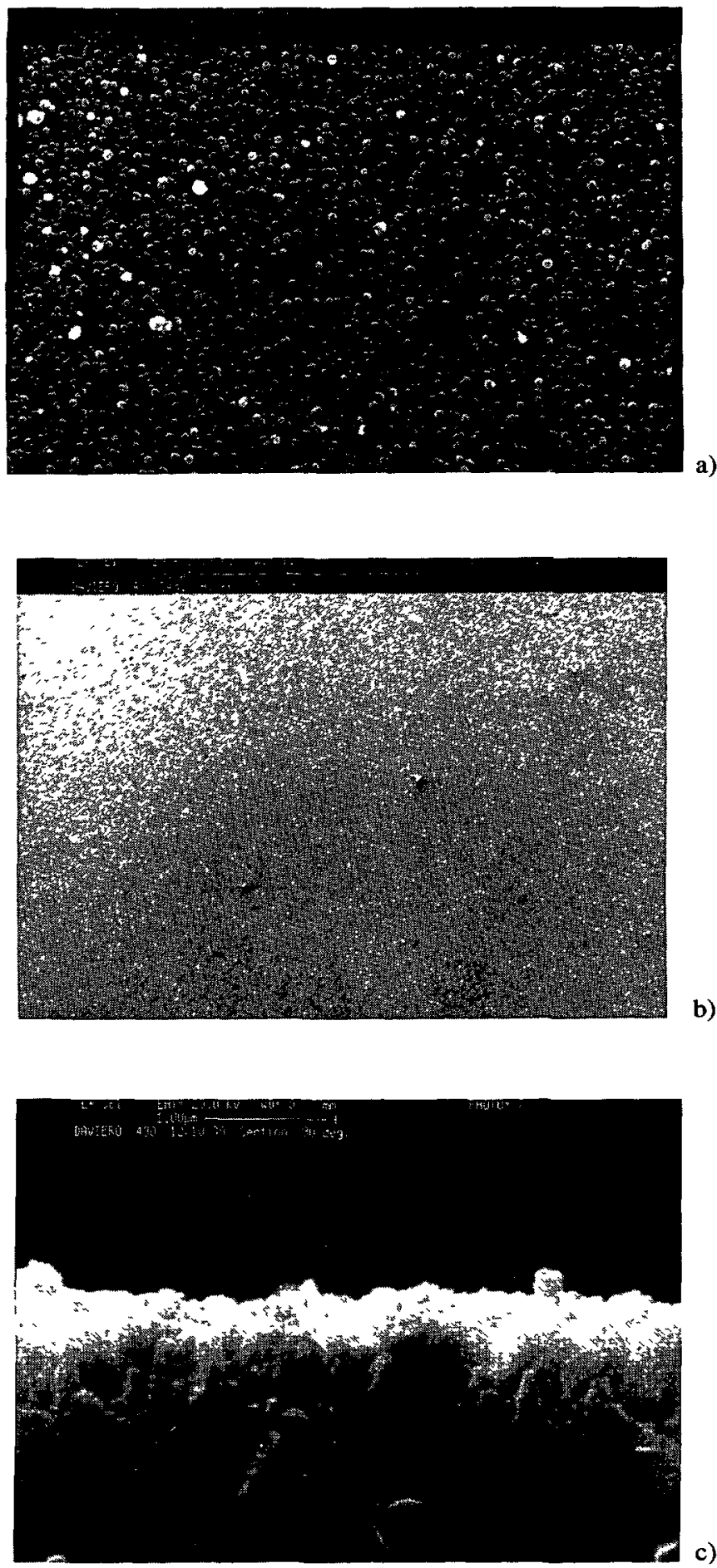

Photos 1. - a) $\mathrm{Al} / \mathrm{P}=0,91$; b) $\mathrm{Al} / \mathrm{P}=3,00$; c) Observation transversale des couches minces. [a) $\mathrm{Al} / \mathrm{P}=0.91$; b) $\mathrm{Al} / \mathrm{P}=3.00$; c) Thin film cross-section.] 
3.2.2 Spectroscopie infrarouge. - La figure 2 reporte les spectres infrarouge obtenus pour les mêmes dépôts. Dans tous les cas, on retrouve les bandes d'absorption caractéristiques des groupements $\mathrm{AlO}_{4}$ et $\mathrm{PO}_{4}$. Ces bandes, très larges, sont caractéristiques d'un matériau amorphe. Pour le spectre de l'échantillon le plus riche en aluminium, la bande d'absorption très large a basse fréquence confirme la présence d'alumine.

3.2.3 Diffraction des rayons $X$. - Dans tous les cas, ces couches ne présentent sur les diffractogrammes de RX qu'un large anneau de diffusion caractéristique de l'état amorphe.

3.3 ETUDE DU RECUIT DES COUCHES MINCES. - Pour essayer de mieux caractériser ces dépôts, des recuits ont été effectués sur des échantillons de composition $\mathrm{Al} / \mathrm{P}$ comprise entre 0,7 et 3,4. Leur évolution a été suivie en fonction de la température, de la durée de recuit et de leur environnement, à l'air ou sous vide.

La recristallisation des dépôts a été suivie par diffraction des $\mathrm{RX}$. La température de cristallisation est dans tous les cas supérieure à $700^{\circ} \mathrm{C}$ et augmente avec la concentration en aluminium. Pour les couches riches en aluminium $(\mathrm{Al} / \mathrm{P}>1,5)$ la cristallisation n'est pas amorcée à $900^{\circ} \mathrm{C}$. Ceci serait en accord avec la présence prépondérante d'alumine dans ces dépôts. Dans tous les cas où une cristallisation apparaît, il y a formation de la phase tridymite du phosphate d'aluminium et non de sa forme berlinite. Ce résultat confirme des études précédentes montrant que la tridymite $\mathrm{AlPO}_{4}$ ne s'obtenait facilement qu'à partir de phosphates hydratés ou amorphes [2].

Les couches recuites sous vide ont tendance à se craqueler. Recuites à l'air, elles présentent un état de surface très lisse en dessous de $800^{\circ} \mathrm{C}$, photo $2 \mathrm{a}$, tandis qu'au-dessus de cette

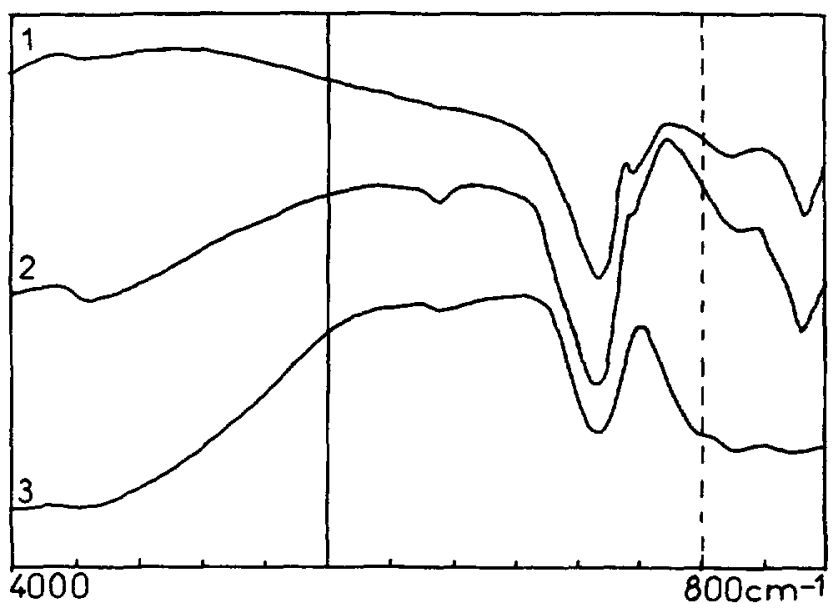

Fig. 2. - Spectres infrarouge des couches minces de composition : (1) $\mathrm{Al} / \mathrm{P}=\mathbf{0 , 8 1}$; (2) $\mathrm{Al} / \mathrm{P}=$ 0,86 ; (3) $\mathrm{Al} / \mathrm{P}=3,00$. A gauche du trait central, pas en énergie de $400 \mathrm{~cm}^{-1}$; à droite : $200 \mathrm{~cm}^{-1}$

[Infrared spectra of some aluminium phosphate thin films with: (1) $\mathrm{Al} / \mathrm{P}=0.81$; (2) $\mathrm{Al} / \mathrm{P}=$ 0.86 ; (3) $\mathrm{Al} / \mathrm{P}=3.00$. From the middle line, step range $=400 \mathrm{~cm}^{-1}$ on the left side and $200 \mathrm{~cm}^{-1}$ on the right one.] 


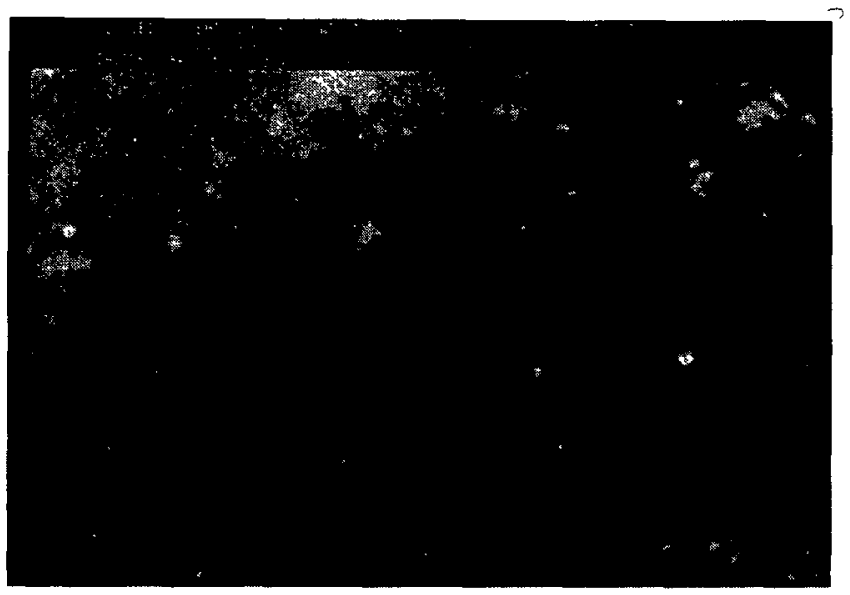

a)

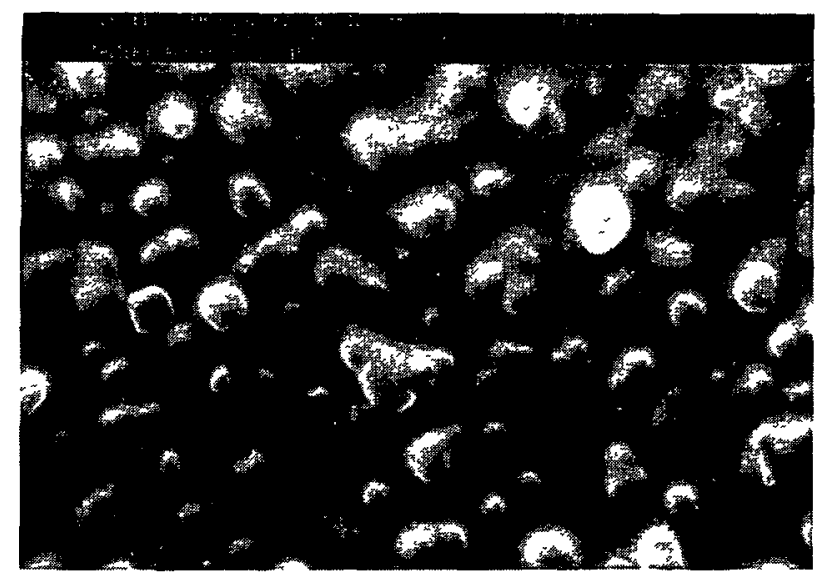

b)

Photos 2. - a) Recuit à $T<800^{\circ} \mathrm{C}$; b) Recuit à $T>800^{\circ} \mathrm{C}$.

[a) Annealed thin film at $T<800^{\circ} \mathrm{C}$. b) Annealed thin film at $T>800^{\circ} \mathrm{C}$.]

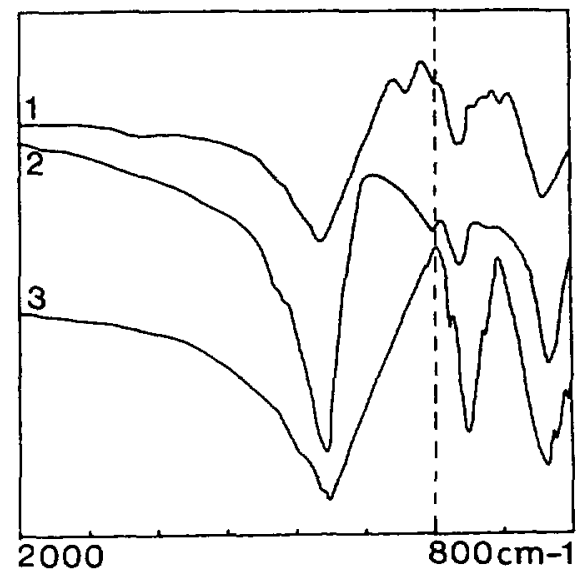

Fig. 3. - Spectres infrarouge: (1) tridymite, (2) couche mince après recuit, (3) berlinite. [Infrared spectra : (1) tridymite, (2) annealed thin film, (3) berlinite.] 
température apparaissent de petites figures de croissance, photo $2 \mathrm{~b}$. D'autre part, les dosages effectués sur des échantillons recuits à l'air et à $850^{\circ} \mathrm{C}$ montrent que les dépôts excédentaires en oxygène ont tendance à perdre cet excès au cours du recuit. De plus, pour les couches dont le rapport Al/P est voisin de 1 les recuits entraînent des modifications de leur composition qui se rapproche de la stœchiométrie $\mathrm{AlPO}_{4}$. Par contre, les couches riches en aluminium ne présentent pas de modification notable. Ces résultats sont en accord avec l'hypothèse de formation de deux types de chaînes [O-Al-O-P $]_{n}$ et $[\mathrm{O}-\mathrm{Al}-\mathrm{O}-\mathrm{Al}]_{n}$.

Enfin les spectres IR des dépôts recuits, riches en phosphore $(\mathrm{Al} / \mathrm{P}<1)$, présentent des bandes d'absorption plus étroites et mieux résolues qui sont très comparables à celles enregistrées pour la forme $\mathrm{AlPO}_{4}$ tridymite (Fig. 3). Ceci est en bon accord avec les résultats de diffraction des RX.

Ces couches étant bien caractérisées en fonction des conditions de dépôt et de recuit, il était intéressant de connaître ensuite leurs caractéristiques électriques en vue d'une éventuelle utilisation.

3.4 CARACTÉRISATION ÉleCTRIQue Des COUCHES minCES. - Nous présentons ici les résultats obtenus en courant continu et en courant alternatif. Nous essaierons ensuite d'expliquer le comportement électrique de ces couches minces.

3.4.1 Mesures électriques en courant continu : conductivité $\sigma_{\mathrm{DC}}$ - - Dans ce cas, la conductivité électrique varie en fonction de l'inverse de la température, et obéit à la loi :

$$
\sigma_{\mathrm{DC}}=\sigma_{0} \exp \left(-W_{2} / k T\right)
$$

où $W_{2}$ est l'énergie potentielle des sites responsables de la conduction. Nous avons reporté dans le tableau III les résultats expérimentaux obtenus à $298 \mathrm{~K}$ pour trois échantillons de composition voisine de ceux déjà étudiés plus haut.

Les valeurs de $\sigma_{\mathrm{DC}}$ pour les couches minces sont nettement plus élevées que celle du matériau massif $\left(\sigma_{\mathrm{DC}} \approx 10^{-22}(\Omega \mathrm{cm})^{-1}\right)$ et cela d'autant plus que le rapport Al/P s'écarte de 1 . Avant d'essayer d'interpréter ces résultats, nous présenterons les mesures électriques effectuées en courant alternatif.

Tableau III. - Résultats des mesures de conductivité électrique en courant continu.

[Experimental results obtained under direct current.]

\begin{tabular}{|c|l|l|c|}
\hline $\mathrm{Al} / \mathrm{P}$ & $\mathrm{O}_{\text {theor }} / \mathrm{O}_{\exp }$ & $W_{2}$ & $\sigma_{\mathrm{DC}}(\Omega \mathrm{cm})^{-1}$ \\
\hline 0,84 & 0,87 & 0,08 & $10^{-10}$ \\
0,91 & 0,88 & 0,10 & $4,0 \times 10^{-11}$ \\
0,81 & 1,00 & 0,09 & $2,1 \times 10^{-10}$ \\
3,00 & 0,946 & 0,13 & $7,5 \times 10^{-10}$ \\
\hline
\end{tabular}

3.4.2 Mesures électriques en courant alternatif : conductivité $\sigma_{\mathrm{AC}}$ - Comme on peut le voir dans un exemple donné en figure 4 , les variations de conductivité, $\sigma_{A C}$, en fonction de la fréquence $\omega$ présentent deux domaines. Ceci nous permet de supposer que $\sigma_{\text {AC }}$ peut s'exprimer sous la forme :

$$
\sigma_{\mathrm{AC}}=\sigma_{\mathrm{DC}}+\sigma_{1}(\omega)
$$




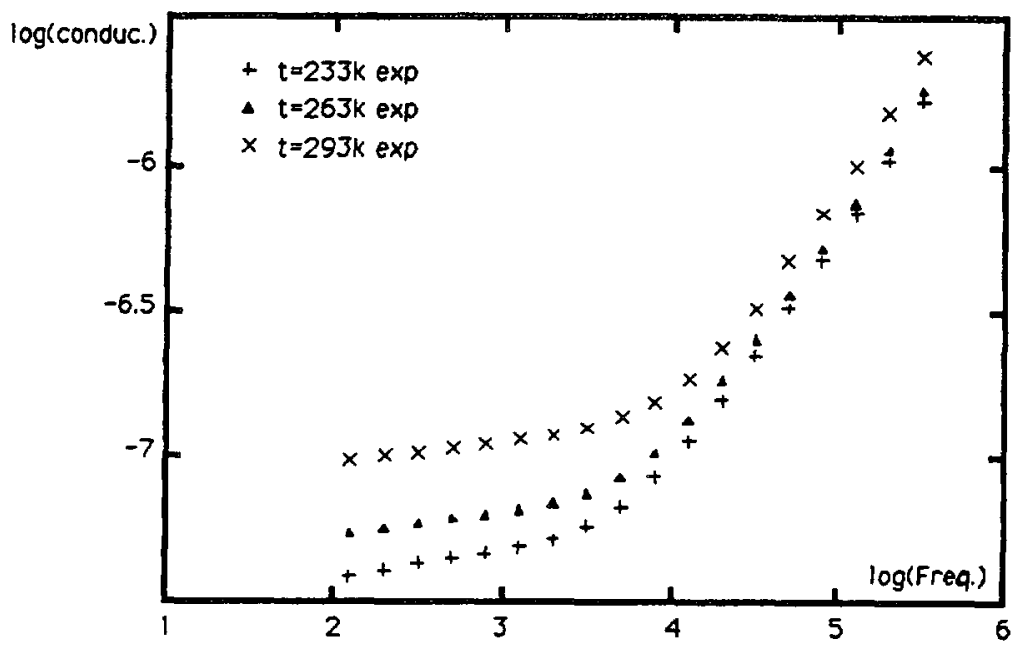

Fig. 4. - Couche mince : $\mathrm{Al} / \mathrm{P}=3,0 ; \mathrm{O}_{\text {théd }} / \mathrm{O}_{\text {exp }}=0,95$. Evolution de $\log$ (cond.) $=f$ (log (fréq.)) en fonction de $T$.

[The evolution of $\log$ (cond. $)=f\left(\log\right.$ (freq.) ) versus $T$ for $\mathrm{Al} / \mathrm{P}=3.0$ and $\mathrm{O}_{\text {theo }} / \mathrm{O}_{\text {exp }}=0.95$ thin film.]

où $\sigma_{1}(\omega)$ représente la partie réelle de la conductivité de polarisation. La variation de cette dernière valeur en fonction de la fréquence suit une loi de la forme :

$$
\sigma_{1}(\omega)=A(T) \omega^{s}
$$

où la variation de $s$ en fonction de la température, si elle obéit à la loi de Long [3] comme c'est le cas, permet de calculer la barrière de potentiel séparant deux sites intervenant dans la conduction, $W_{\mathrm{M}}$, et le nombre de porteurs de charge, $N$. Nous avons regroupé dans le tableau IV les différentes valeurs obtenues :

Tableau IV. - Résultats des mesures électriques en courant alternatif.

[Experimental results obtained under alternating current.]

\begin{tabular}{|c|c|c|c|c|c|}
\hline $\mathrm{Al} / \mathrm{P}$ & $\mathrm{O}_{\text {theor }} / \mathrm{O}_{\exp }$ & $T(\mathrm{~K})$ & $s$ & $W_{\mathrm{M}}$ & $N$ \\
\hline 0,81 & 1,00 & $213-303$ & $0,93-0,90$ & 2,00 & $3,1-3,9 \times 10^{22}$ \\
0,91 & 0,88 & $208-301$ & $0,92-0,88$ & 1,80 & $2,0-2,2 \times 10^{22}$ \\
0,86 & 0,84 & $223-303$ & $0,81-0,71$ & 0,98 & $2,0-2,4 \times 10^{22}$ \\
3,33 & 0,86 & $194-293$ & $0,83-0,80$ & 1,10 & $5,2-5,1 \times 10^{22}$ \\
\hline
\end{tabular}

Sur la figure 5 nous avons reporté l'évolution de la barrière d'énergie, $W_{\mathrm{M}}$, en fonction du rapport $\mathrm{O}_{\text {théor }} / \mathrm{O}_{\text {exp }}$. On observe que cette valeur augmente quand 1'excès d'oxygène diminue. 


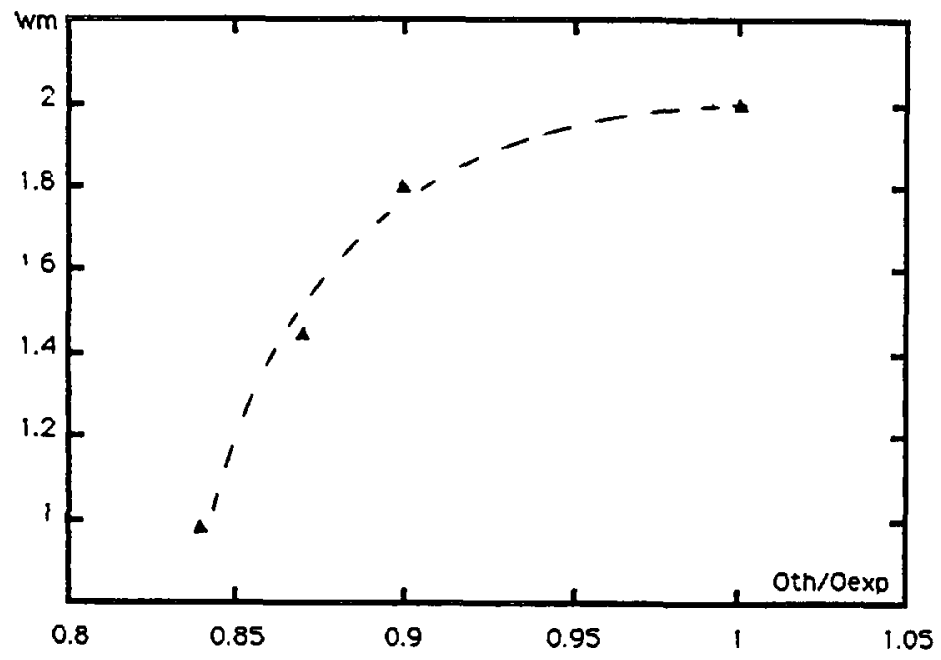

Fig. 5. - Evolution de $W_{M}$ en fonction du rapport $\mathrm{O}_{\text {theo }} / \mathrm{O}_{\text {exp }}$ pour les couches minces avec $\mathrm{Al} / \mathrm{P}<1$.

[Evolution of $W_{M}$ versus the $\mathrm{O}_{\text {theo }} / \mathrm{O}_{\text {exp }}$ ratio of different thin films with $\mathrm{Al} / \mathrm{P}<1$. ]

Nous avons pu expliquer ces différents résultats en utilisant le modèle CBH (Correlated Barrier Hopping) proposé par Pike [4] et développé par Elliott [5]. Dans ce cas, le transfert électronique est attribué à l'activation thermique qui permet de franchir la barrière de potentiel entre deux sites de conduction, $W_{M}$. Dans ce cas, l'abaissement de la barrière d'énergie est favorisée par la rupture de liaisons Al-O-P, conduisant à la formation de liaisons pendantes, $\mathrm{Al}-\mathrm{O}$ et $\mathrm{P}-\mathrm{O}$. La conduction électronique est alors assurée par sauts simultanés de paires électroniques entre les sites de conduction. Ce modèle explique donc bien la diminution de $W_{\mathrm{M}}$ avec l'excès d'oxygène, car cet excès doit s'accompagner d'une multiplication des défauts structuraux liés aux liaisons pendantes.

Dans la dernière partie, nous présenterons les premiers résultats d'une approche structurale de ces couches minces effectuée par spectroscopie d'absorption des rayons $\mathrm{X}$.

3.5 APPROCHE STRUCTURALE DES COUCHES MINCES. - Cette étude a permis de caractériser l'environnement local des atomes d'aluminium et de phosphore. Les mesures ont été effectuées en utilisant le rayonnement synchrotron de l'anneau de stockage SACO du LURE, Orsay, aux seuil $\mathrm{K}$ de l'aluminium et du phosphore.

$\mathrm{Au}$ seuil $\mathrm{K}$ du phosphore, les résultats sont limités car ils ne confirment que l'environnement tétraédrique de l'atome de phosphore par les atomes d'oxygène. L'étude EXAFS (Extended Xray Absorption Fine Structure) montre toutefois un léger allongement des liaisons $\mathrm{P}-\mathrm{O}$ quand on passe de la berlinite, $\mathrm{AlPO}_{4}$, aux couches minces. De plus, il semble que l'environnement $\mathrm{du}$ phosphore soit très comparable à celui de la tridymite, $\mathrm{AlPO}_{4}$, expliquant ainsi la recristallisation sous cette forme observée lors des recuits.

Pour le seuil K de l'aluminium, c'est l'étude XANES (X-ray Absorption Near Edge Structures) qui apporte plus de renseignements. Nous avons pu ainsi démontrer l'existence dans nos couches d'atomes d'aluminium en sites tétraédriques $\mathrm{AlO}_{4}$ et octaédriques $\mathrm{AlO}_{6}$. En effet, les spectres XANES des couches minces présentent à la fois les structures d'absorption de la berlinite $\left(\mathrm{AlO}_{4}\right)$ et celle de l'alumine $\alpha\left(\mathrm{AlO}_{6}\right)$ (Fig. 6). De plus, il semble que la 


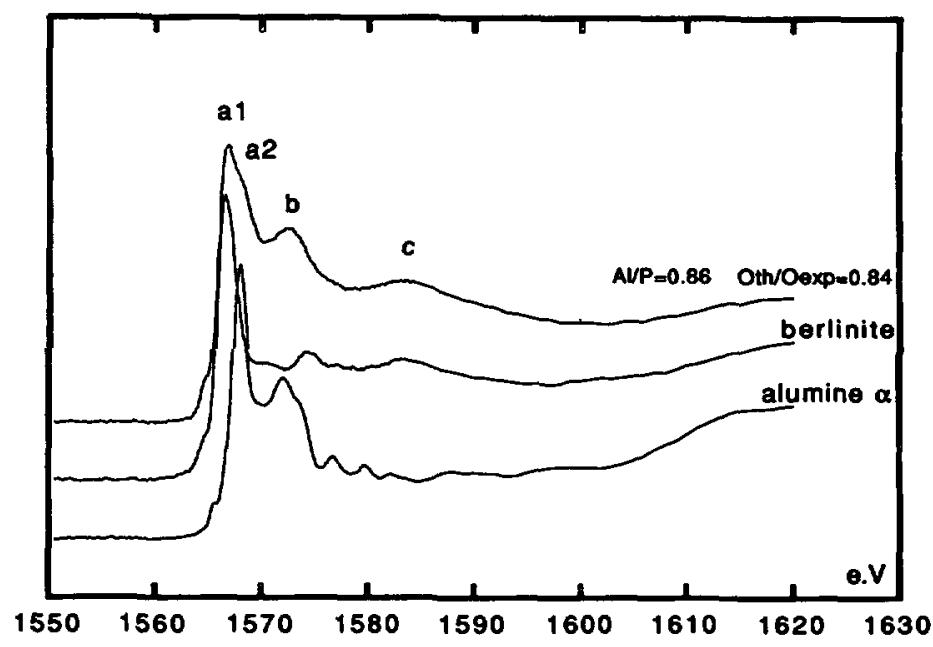

Fig. 6. - Spectres XANES de la couche mince de rapport $\mathrm{Al} / \mathrm{P}=0,86$ avec les spectres des deux références cristallisées.

[XANES spectra for the thin film with $\mathrm{Al} / \mathrm{P}=\mathbf{0 . 8 6}$, in comparison with the crystallized references.]

proportion en $\mathrm{AlO}_{6}$ soit directement liée à l'excès d'oxygène pour les couches riches en phosphore $(\mathrm{A} 1 / \mathrm{P}<1)$. D'autre part, les spectres des dépôts riches en aluminium sont très voisins de ceux obtenus pour une alumine amorphe.

Ces résultats confirment donc la coexistence dans ces couches minces d'enchaînements [O-Al-O-P $]_{n}$ que l'on trouve dans la forme tridymite, $\mathrm{AlPO}_{4}$, d'une part, et d'autre part, d'enchaînements de type [O-Al-O-Al $]_{n}$ que l'on rencontre dans les alumines amorphes avec $\mathrm{Al}$ en sites tétraédrique et octaédrique.

\section{Conclusion.}

Dans ce travail, nous avons tout d'abord défini des conditions de dépôt de couches minces de phosphate d'aluminium sur substrat semi-conducteur à l'aide du procédé « pyrosol ». Ces films minces ont été réalisés pour un rapport variable des précurseurs TBP et AAA en solution. Ils conduisent à un très large éventail de composition chimique en fonction de la température du substrat et des solutions utilisées. Quelle que soit la température de dépôt, ces couches sont amorphes et peuvent être recristallisées par recuit sous forme $\mathrm{AlPO}_{4}$ tridymite.

L'approche structurale, réalisée par spectroscopie d'absorption des $R X$, a confirmé la coexistence de deux types d'enchaînements [O-Al-O-P $]_{n}$ et $[\mathrm{O}-\mathrm{Al}-\mathrm{O}-\mathrm{Al}]_{n}$ dans ces dépôts avec des atomes d'aluminium en site tétraédrique et octaédrique. On peut donc supposer que la structure de ces couches minces est constituée par deux types d'agrégats :

- le premier, basé sur la structure $\mathrm{AlPO}_{4}$ tridymite, recristalliserait sous cette forme lors de recuits ;

- le second, basé sur la structure d'une alumine amorphe, deviendrait largement majoritaire pour les dépôts riches en aluminium et ne recristalliserait pas jusqu'à $950^{\circ} \mathrm{C}$ environ.

Les mesures de conductivité électrique en courant continu ont montré que ces dépôts présentent une résistivité élevée mais qui est cependant nettement plus faible que celle du matériau massif. Les mesures de conductivité en courant alternatif ont permis ensuite, en 
s'appuyant sur des travaux développés par Elliott, de préciser les sites intervenant dans la conduction qui sont, dans ces dépôts, les atomes d'oxygène non pontants. Pour améliorer les caractéristiques électriques, il faut donc éliminer au maximum les défauts structuraux et donc l'excès d'oxygène en se rapprochant de la stœchiométrie $\mathrm{AlPO}_{4}$. Pour cela on peut envisager des modifications de synthèse, en particulier utiliser un gaz vecteur inerte, d'autres précurseurs, d'effectuer des recuits, etc.

Pour terminer, on peut dire que la technique «pyrosol » est maintenant bien maîtrisée au laboratoire. Nous avons donc commencé à appliquer cette technique de dépôt à d'autres compositions, phosphate de gallium et arséniate d'aluminium, et à d'autres substrats semiconducteurs, en particulier GaAs.

\section{Bibliographie}

[1] Daviero S., Avinens C., Ibanez A., Cambié C., Maurin M., Phimippot E., Dépôt de films minces de phosphate d'aluminium par le procédé pyrosol, Le Vide, Les Couches Minces 250 (1990) 23.

[2] Allaf K., Rouanet A., Relations entre les phases de l'orthophosphate d'aluminium $\mathrm{AlPO}_{4}, \mathrm{High}$ Temp.-High Pressures 10 (1978) 591.

[3] Long A. R., Frequency dependent loss in amorphous semiconductors, Adv. Phys. 31 (1982) 553.

[4] PIKe G. E., Ac conductivity of scandium oxide and a new hopping model for conductivity, Phys. Rev. B 6 (1972) 1572.

[5] Elliott S. R., Ac conduction in amorphous chalcogenide and pnictide semiconductors, Adv. Phys. 36 (1987) 135. 